\title{
«Le parole sono tenere cose»: spunti per un'analisi linguistica e strutturale di La casa in collina di Cesare Pavese
}

\author{
SILVIA ZANGRANDI \\ IULM Milano \\ silvia.zangrandi@iulm.it
}

\section{Riassunto}

Lo studio intende offrire una lettura su più piani di La casa in collina a partire dall'abolizione del distacco prospettico dell'autore dalla sua materia e da una sua immersione nel flusso degli avvenimenti, per passare poi all'esigenza di analisi e di autoanalisi e alla sostituzione del principio di causalità con quello relativistico che implica una concezione dell'esistenza come pura possibilità. Nell'assetto generale del racconto troviamo momenti narrativi e momenti di riflessione e il punto di vista nella conduzione del racconto non è affidato unicamente al narratore, ma viene spesso filtrato attraverso le parole dei personaggi chiamati in scena. La realtà storico-politica qui rappresentata non impedisce di dar vita a situazioni lirico-descrittive e a momenti intensamente dolorosi che sollecitano l'emozionalità del lettore. La presenza imponente del dialogo, mezzo mimetico per antonomasia, dà a Pavese la possibilità di ricreare il parlato popolare: tramite l'uso di dialettismi, di ridondanze morfosintattiche, di pleonasmi, del che polivalente egli riproduce la parlata informale e quotidiana e riferisce con autenticità le parole del personaggio.

Parole chiave: Narratività, mimesi, dialogo, lingua, parlato popolare, dialettismi, Pavese, $L a$ casa in collina.

«Le parole sono tenere cose»: sketches for a linguistic and structural analysis of

Cesare Pavese's La casa in collina

\begin{abstract}
The paper aims to provide a multi-layered reading of La casa in collina. It focuses on the abolition of the perspective distance of the author from his matter which leads to his immersion in the flow of events, to the need of analysis and self-analysis and to the substitution of the principle of causality with a relativistic one involving a conception of existence as mere chance. In the general organization of the story, narrative moments are followed by moments of reflection. The point of view in the storytelling is not given solely by the narrator but is
\end{abstract}


often filtered through the words of the characters. The historical and political reality represented in the novel does not preclude lyrical-descriptive situations and painful scenes which call upon the emotions of the reader. The overwhelming presence of dialogue, which is by definition a mimetic medium, gives Pavese the possibility to recreate people's popular speech: through the use of dialectalism, morphosyntactic redundancy, pleonasms, non-standard relative clauses he reproduces informal everyday speech and reports the characters' words with authenticity.

Key words: Narrativity, mimesis, dialogue, language, «parlato popolare», dialectalism, Pavese, La casa in collina.

Zangrandi, Silvia. 2011. «Le parole sono tenere cose»: spunti per un'analisi linguistica e strutturale di La casa in collina di Cesare Pavese. Cuadernos de Filología Italiana, $\mathrm{n}^{\circ}$ extraordinario: pags. 377-389.

L'evidente correlazione tra contenuto narrativo e vita dell'autore, che appare durante la lettura del romanzo di Cesare Pavese La casa in collina (1948), non deve essere usata come modalità esclusiva per un corretto approccio al testo. Infatti, oltre alla coincidenza tra autore e narratore, emerge anche quella col protagonista Corrado: questa identificazione è rintracciabile attraverso le funzioni morfologiche del monologo e del dialogo e tramite un bilanciamento che si muove tra le capacità autocritiche della coscienza-conoscenza del narratore e quella del protagonista che diventa lo schermo riflettente dell'esperienza dell'autore: ci troviamo di fronte a un io autobiografico riconoscibile che va incontro a una realtà storica dolorosa e pressante. L'autore pare convincerci che la situazione di soggettività di chi scrive non sia una schiavitù, ma l'accettazione di un processo catartico attraverso una scrittura che, con l'intersecarsi dei piani, il moltiplicarsi dei punti di vista, la linearità del linguaggio, si offre agli altri come motivo di crescita e di promozione sociale. Per dirla con Robbe-Grillet, «la soggettività relativa del mio sguardo mi serve precisamente a definire la mia situazione nel mondo. Evito semplicemente di concorrere, io stesso, a fare di questa situazione, una servitù» (Robbe-Grillet 1961: 89). Si riscontra una certa abdicazione del controllo del soggetto sull'oggetto, che sono quasi identificati non nella struttura, ma semanticamente. Assistiamo così all'abolizione del distacco prospettico dell'autore dalla sua materia e a una sua immersione nel flusso degli avvenimenti. La riproduzione letteraria del reale non presenta solo un concatenamento fattuale di natura meccanicistica, ma mostra anche un'esigenza di analisi e di autoanalisi e la sostituzione del principio di causalità con quello relativistico che implica una concezione dell'esistenza come pura possibilità e che si esplicita nelle dichiarazioni: «siamo al mondo per caso» (Pavese 2007: 46); "perché la salvezza era toccata a me e non a Gallo, non a Tono, non a Cate, non so. Forse perché devo soffrire dell'altro?» (Pavese 2007: 87). Questa possibilità è accompagnata da una natura se non matrigna certo indifferente al divenire del mondo: «la città poteva andare tutta in fiamme e la gente morire. I burroni, le ville e i sentieri si sarebbero svegliati al mattino calmi e uguali» (Pavese 2007: 3); «il sangue sparso era 
assorbito dalla terra. Le città respiravano. Soltanto nei boschi nulla mutava, e dove un corpo era caduto riaffioravano radici» (Pavese 2007: 33); «eppure tutto continuava. Sorgeva il mattino, calava la sera, maturava la frutta» (Pavese 2007: 62). L'aleatorietà della vita, per cui ogni azione umana ne esclude molte altre possibili, pone il narratore di fronte alla realtà non tanto e non solo per registrarla, ma per interpretarla, trasformarla e proporla a chi legge:

noi rispettiamo troppo il nostro mestiere, per illuderci che l'ingegno, l'invenzione, ci bastino. Nulla che valga può uscirci dalla penna e dalle mani se non per un attrito, per urto con le cose e con gli uomini. Libero è solamente chi si inserisce nella realtà e la trasforma, non chi procede tra le nuvole [...] Siamo tutti convinti che solo il mondo e la vita contengono gli spunti, le condizioni di qualunque pagina vera si è scritta o si scriverà (Pavese 1971: 232, 238).

\section{Leggiamo l'apertura del romanzo:}

Già in altri tempi si diceva la collina come avremmo detto il mare o la boscaglia. Ci tornavo la sera, dalla città che si oscurava [...] Per esempio, non vedevo differenza tra quelle colline e queste antiche dove giocai bambino e adesso vivo: sempre un terreno accidentato e serpeggiante, coltivato e selvatico, sempre strade, cascine e burroni. Ci salivo la sera come se anch'io fuggissi il soprassalto notturno degli allarmi (Pavese 2007: 3).

Il segmento retrospettivo che esordisce con «già in altri tempi» è un mezzo di delucidazione e di coinvolgimento psicologico del lettore e unisce il pensiero del narratore a quello del personaggio. La confusione tra passato e presente che si oppone alla mancata differenza tra le colline, la nominalità dell'aspetto paesaggistico «sempre un terreno accidentato e serpeggiante, coltivato e selvatico, sempre strade, cascine e burroni» (Pavese 2007: 3) - non ammettono dubbi sulla paternità del narratore che si identifica con quella del protagonista e guida il filo del racconto con l'introduzione della prima persona, «ci tornavo [...] ci salivo», fino allo sbocco finale in cui si riepiloga il passato, si esprime la situazione del presente e l'incertezza del futuro. Analogamente, alla p. 9 vengono redatti i pensieri del protagonista nati dopo l'incontro con Cate: le pressanti domande diventano specchio riflettente di una situazione passata dove il narratore si fa giudice di se stesso e dove la figura del protagonista raggiunge la più perspicua connotazione proprio attraverso le insistenti domande, i numerosi deittici e l'enfasi dell'anafora «il giovane temerario [...] il giovane $[\ldots]$ questo giovane», fino ad arrivare all'amara conclusione in cui si domanda come abbia potuto gustare e tradire una vita così. La forza drammatica delle domande, la capacità di penetrazione nell'animo del protagonista eclissano la visione oggettiva del narratore per lasciare viva solo quella del personaggio chiamato in causa.

Nel lacerto seguente rimane viva nella sua immediatezza la visione soggettiva dell'attore chiamato in causa: è attraverso questo modo di procedere che emerge con pienezza la forza emotiva della sequenza: 
pensai all'eco dei clamori, al sangue sparso, agli spari. Quanto sangue, mi chiesi, ha già bagnato queste terre, queste vigne. Pensai che era sangue come il mio, ch'erano uomini e ragazzi cresciuti a quell'aria, a quel sole, dal dialetto e dagli occhi caparbi come i miei. Era incredibile che gente come quella che mi vivevano nel sangue e nel chiuso ricordo, avessero anche loro subito la guerra, la ventata, il terrore del mondo. Per me era strano, inaccettabile, che il fuoco, la politica, la morte sconvolgessero quel mio passato. Avrei voluto trovar tutto come prima, come una stanza stata chiusa (Pavese 2007: 109).

Osserva Eleonora Cane: «il dualismo autore-personaggio o quello ancora più complesso autore-personaggio-narratore [è] dualismo che si articola [...] in una sorta di ambiguità oggettiva-soggettiva in cui è difficile distinguere con sicurezza dove l'autore parli per conto suo o per conto del proprio personaggio» (Cane 1969: 15-16). Consideriamo ora quest'altro passaggio:

e se non fosse che la guerra ce la siamo covata nel cuore noialtri - noi non più giovani, noi che abbiamo detto "venga dunque se deve venire» - anche la guerra, questa guerra, sembrerebbe una cosa pulita. Del resto, chi sa. Questa guerra ci brucia le case. Ci semina di morti fucilati piazze e strade. Ci caccia come lepri di rifugio in rifugio. Finirà per costringerci a combattere anche noi, per strapparci un consenso attivo. E verrà il giorno che nessuno sarà fuori della guerra - né $\mathrm{i}$ vigliacchi, né i tristi, né i soli (Pavese 2007: 120-121).

Colpisce qui l'uso iterato del verbo al tempo presente, tempo che, oltre a sottolineare le meditazioni del protagonista, viene usato con l'intento di dare un carattere universale a ciò che dice Corrado, che rivive gli avvenimenti causati dalla guerra, enfatizzati dall'iterazione anaforica del pronome personale $c i$. Il tempo dell'attualità è interrotto da una previsione futura, «finirà $[\ldots]$ verrà $[\ldots]$ sarà», e qui prevale l'idea del distacco tra vicenda narrata e narratore che non rivive quanto racconta, ma lo osserva criticamente («verrà il giorno che nessuno sarà fuori della guerra - né i vigliacchi, né i tristi, né i soli»). Pavese non si limita a filtrare le parole dei personaggi, ma mette in evidenza la progressiva presa di coscienza del narratore e permette lo sdoppiamento psicologico tra il piano in cui il protagonista si fa narratore e quello in cui si fa commentatore e può riflettere su quello che sta raccontando, giungendo spesso a concludere dolorosamente che ora che ha visto cos'è la guerra è convinto che tutti debbano chiedersi perché tanti sono morti, constatando con sofferenza che nessuno è in grado di dare una risposta.

Nell'assetto generale del racconto troviamo momenti narrativi e momenti di riflessione e il punto di vista nella conduzione del racconto non è affidato unicamente al narratore, ma viene spesso filtrato attraverso le parole dei personaggi chiamati in scena in modo da provocare una rifrazione continua dell'ottica conduttrice. A volte fa capolino l'imparzialità della terza persona, ma subito cede il passo all'ingerenza perentoria della prima. Si vedano ad esempio le prime pagine del capitolo $\mathrm{X}$ : la tonalità emotiva della sequenza ribadita dalle qualità connotanti di aggettivi, verbi e avverbi (soprattutto di tempo) che preludono la lunga serie di avvenimenti tragici e di distruzioni, dà vita a una progressiva assimilazione tra narratore e prota- 
gonista; è da ciò che scaturisce l'intervento della prima persona «rientrando in collina $[\ldots]$ avrei voluto esser radice, essere verme e sprofondare sottoterra» (Pavese 2007: 52). Corrado si trova a vivere all'interno del discorso narrativo le sensazioni del narratore, mettendo in atto un annullamento del narratore nel proprio oggetto, cioè il protagonista del romanzo; dopo la manifestata volontà del primo pensiero, "avrei voluto», si susseguono in serie concitata gli altri pensieri caratterizzati da frasi brevi e irruenti separate da punti fermi che si concludono con la forza dell'anafora data dal verbo "preferivo». Subito dopo il filo emozionale è spezzato dal cambiamento del soggetto e dalla presenza del passato remoto «diede» che ripristina il flusso diegetico della narrazione.

In altri momenti si nota questa identificazione ideologico-affettiva tra narratore e protagonista; nell'iterazione del verbo al tempo imperfetto fa capolino l'intervento del passato remoto che opera una specie di passaggio metaforico del punto di vista. Prendiamo ad esempio le pagine iniziali del cap. XIII: il passo è orchestrato sulla reiterazione dei verbi al tempo imperfetto che mostrano una scena vissuta e sofferta, estrapolata da un tessuto memoriale triste, reso efficacemente da aggettivi e sostantivi altamente pertinenti: «allibite $[\ldots]$ paurose $[\ldots]$ macerie $[\ldots]$ rovine $[\ldots]$ disagi $[\ldots]$ cose bestiali $[\ldots]$ congiure $[\ldots]$ torture $[\ldots]$ morti freschi»; dalla visione del «grosso topo tranquillo nel sole» (p. 68) che, a differenza degli uomini, non ha paura di niente e dall'affermazione «la città si era fatta più selvaggia dei miei boschi». Appare improvvisamente il passato remoto «m'accorsi», come se la mente del protagonista solo in quel momento riuscisse a mettere a fuoco una situazione sofferta e ineluttabile che «afferrava le viscere». Il flusso narrativo continua sempre condotto dai verbi al tempo imperfetto e neppure le battute dialogiche riescono ad animare le tristi scene evocate, tutto è assimilato alla morte e alla distruzione, perfino i fiori e le piante del frutteto «squallido e secco» (Pavese 2007: 70); la visione si conclude con la forza d'urto del passato remoto «venne un gran vento e lo spazzò» (p. 70). È con questa metafora del vento che si esplicita la progressiva assimilazione tra il narratore e Corrado il quale vive all'interno del discorso narrativo il turbamento e le sensazioni evocate dall'autore-narratore.

Nell'organizzazione del romanzo i personaggi non sono costruiti dall'esterno da un deus ex machina, ma presentati nel loro aspetto multiforme, dando a tutta la narrazione una forma plurifocalizzata attraverso l'adozione di punti di vista dei diversi personaggi. il compito di denunciare il comportamento di chi comanda è affidato alla vecchia di Cate: «se si mettessero d'accordo. Tanto tra loro non si mangiano. Chi va di mezzo siamo noi» (Pavese 2007: 22), oppure quello di chi non si mette in gioco: «venite a dirlo a chi lavora. Per chi ha la pagnotta e può stare in collina, la guerra è un piacere. Sono la gente come voi che ha portato la guerra» (Pavese 2007: 50). Nello stesso modo il narratore demanda agli avventori dell'osteria il compito di considerare il comportamento dello stato, dei tedeschi, dei fascisti: sono questi i temi e le parole dello stesso narratore. «I fascisti resistono [...] l'hanno capita tra loro gerarchi che la storia puzzava, e allora corrono dal re e gli fanno: "senti: ci devi mettere a riposo. Tu intanto continui la guerra, gli italiani si sfogano, si fiaccano il collo, e noi domani ritorniamo a darti una mano"» (Pavese 2007: 36, 41). 
Un'attenzione particolare va data al personaggio di Cate che, simile a un ventriloquo che fa parlare gli attori, viene eletta dal narratore quale voce della sua coscienza: Cate costringe Corrado a porsi criticamente di fronte al suo comportamento immobilista: «tu non fai niente?» (Pavese 2007: 22); «sei buono così, senza voglia. Lasci fare e non dai confidenza. Non hai nessuno, non ti arrabbi nemmeno [...] tu hai paura, Corrado [...] sei tu [...] tu vivi così» (p. 31); «per non farle, ti rendi le cose impossibili» (Pavese 2007: 46); «tu te ne infischi [...] ma hai ragione. Non hai mai visto far la fame né bruciare casa tua» (Pavese 2007: 60). In questo modo il lettore assiste alla coraggiosa e spietata confessione del narratore che non si sottrae dal mettere in luce la sua codardia, anzi la contrappone al coraggio degli altri. Attraverso i dialoghi e i comportamenti delle persone semplici come gli avventori dell'osteria, il lettore è portato a riflettere e a comprendere il comportamento del protagonista. L'ottica di Corrado, pur essendo quella privilegiata, lascia spazio ai punti di vista dei diversi personaggi che non sono sempre soggiogati dal raggio visivo del protagonista, ma presentati nel loro modo di pensare e di agire secondo la loro personale capacità conoscitiva. Il rapporto dell'autore con i suoi personaggi si configura come una relazione con soggetti autonomi e non manovrati dall'alto. Pavese stesso ebbe a dire: «i personaggi sono per lui [Pavese] un mezzo, non un fine. I personaggi gli servono semplicemente a costruire delle favole intellettuali il cui tema è il ritmo di ciò che accade [...] La trasfigurazione angosciosa della campagna e della vita quotidiana nella Casa in collina [...] I personaggi [...] sono del tutto sommari, sono nomi e tipi, non altro: stanno sullo stesso piano di un albero, di una casa, di un temporale o di un'incursione aerea» (Pavese 1971: 288). In La casa in collina non ci sono personaggi a tutto tondo ma ci «sono nomi e tipi» che il lettore scopre piano piano: prendiamo come esempi Cate e Fonso. La prima ci appare inizialmente come il narratore la ricorda ai tempi della sua prima giovinezza «una figliola beffarda e disoccupata, magra e un poco goffa, violenta» (Pavese 2007: 10); poche pagine dopo incontriamo la Cate del presente: «sotto la luna la vedevo bene. Era la stessa ma sembrava un'altra. Parlava sicura di sé [...] era vestita di una gonna corta, da campagna» (Pavese 2007: 21); "Cate era seria, era padrona, Cate capiva come e meglio di me» (Pavese 2007: 32). Piano piano ci troviamo di fronte un personaggio che non è solo un nome e un tipo, ma è una donna che il narratore ammira per «la sua dolcezza, la fermezza» (Pavese 2007: 44). Fonso viene dipinto in tutta la sua irruenza di ragazzo coraggioso, furbo, capace di destreggiarsi in una situazione pericolosa e difficile: «Fonso era un ragazzo, non aveva diciott'anni [...] era cinico, burlone, si accendeva di colpo» (Pavese 2007: 24). Con il suo «sorriso sornione [...] strizzò l'occhio e tirò fuori un permesso bilingue da fattorino e guardiano notturno»» (Pavese 2007: 65); «Fonso correva le montagne e rischiava la pelle» (Pavese 2007: 95). E infine il narratore commenta con rammarico che mai lui aveva «avuto il sangue ardito di Fonso» (Pavese 2007: 95).

Questi personaggi che danno «il ritmo di ciò che accade» non ci sembrano assolutamente sommari e, anche se sono tratteggiati come si farebbe per un albero o una casa, appaiono in tutta la loro umanità e si presentano come «trasfigurazione angosciosa della vita quotidiana»; l'autore ci propone un tratto che piano piano si allarga e infine si svela e, come dice Roland Barthes, «più la distanza sintagmatica fra 
due informazioni convergenti è grande, più è abile il racconto; la bravura consiste nel saper dosare un'impressione: bisogna che il tratto passi leggermente, come se non contasse che resti inosservato e che però, nato più avanti sotto un'altra forma, costituisca già un ricordo» (Barthes 1973: 26-27).

Spesso la funzione di descrivere i fatti viene affidata al dialogo: questo mezzo mimetico è lo strumento più idoneo a conferire potere autonomo al personaggio. Prendiamo ad esempio il cap. VI dove il dialogo tra il narratore e Cate assume un ritmo febbrile e una tenera intensità: le battute dialogiche si fanno pressanti ed evidenziano le personalità dei due parlanti: sicura e forte l'una («Cate era seria, era padrona», Pavese 2007: 31), insicura, ansiosa e sofferente l'altra (Cate così parla di Corrado: «adesso soffri e mi fai pena [...] vivi solo col cane. Mi fai pena», Pavese 2007: 31). Con frequenza i molti dialoghi presenti nel romanzo servono non solo a dar voce a propositi, a descrivere fatti avvenuti, ma anche a mettere in luce il carattere, la personalità dei personaggi e a riferire nel modo più autentico le loro affermazioni per mezzo della parlata quotidiana: «parola - diceva - se mi chiamano sotto, salta in aria il distretto» (Pavese 2007: 24); «questi scemi d'inglesi - dicevano - non lo sanno che basta un'incursione per guastare il lavoro clandestino di un mese? Quando brucia la casa ci tocca scappare anche noi» (Pavese 2007: 49). Sono moduli linguistici vistosamente colloquiali che caratterizzano la semplicità del personaggio in questione.

In diversi punti del racconto viene ricreato il parlato popolare tramite l'uso di dialettismi, di ridondanze morfosintattiche, di pleonasmi, del che polivalente: grazie a essi Pavese riproduce la parlata informale e quotidiana e riferisce con autenticità le parole del personaggio. Di seguito diamo alcuni esempi di colloquialismi, ridondanze, che polivalente, uso del verbo al modo indicativo anziché congiuntivo, uso di mica:

- «Faceva strano vedere i soldati» (Pavese 2007: 16); «per poter pagare, comandavo del vino» (Pavese 2007: 26); "com'è che si chiama quella storta che vuole sposarti?» (Pavese 2007: 28); «ma neh, professore, che tempi» (Pavese 2007: 59); «la madre lavora e il bambino viene su come può» (Pavese 2007: 86)

- «Ancora ci pensi a quei tempi?» (Pavese 2007: 21); «doveva averci colleghi a Torino» (Pavese 2007: 24); «Cate, ci hai proprio la passione» (p. 38); «le disgrazie c'è chi se le cerca» (Pavese 2007: 70)

- «veniva il momento che salivo ormai solo» (Pavese 2007: 3); «arrivai sulla strada che pensavo alla guerra» (Pavese 2007: 20); «un giorno che entrai in cucina» (Pavese 2007: 54); «viviamo in tempi che nessuno è sicuro» (Pavese 2007: 73);

- «se arrivava il giorno prima, vedeva i cadaveri» (Pavese 2007: 76); «se venivano a Chieri, eravamo già morti» (Pavese 2007: 92); «sapevo che prima di domani non arrivavo a casa» (Pavese 2007: 116).

- «Non sei mica fascista?» (Pavese 2007: 22); «non è mica cattivo» (Pavese 2007: 45); «non sei mica di leva» (Pavese 2007: 71); «non è mica la roba» (Pavese 2007: 83). 
Sono abbondanti anche le dislocazioni a sinistra dove «l'elemento dislocato a sinistra sarebbe il frutto della messa a centro d'attenzione del parlante [...] congruente con i caratteri di sintassi egocentrica spesso presenti nel parlato» (Berruto 1998: 66): «quelli la testa non la cambiano» (Pavese 2007: 25); «i massacri li faranno con calma» (Pavese 2007: 58); «mi chiesi se Dino lo mandavano a messa» (Pavese 2007: 80); «Otino non lo trovai» (Pavese 2007:117).

Tuttavia la prosa solo all'apparenza è dimessa e disadorna, la diegesi del racconto infatti è spesso insidiata da situazioni lirico-descrittive e da momenti intensamente dolorosi che sollecitano l'emozionalità del lettore. La descrizione del paesaggio collinare, puntuale e coinvolgente, è resa con un montaggio narrativo fedele al reale, appassionato e commosso, come si evince dalla lettura dei passaggi seguenti:

Preferii soffermarmi su una svolta della salita sgombra di piante, di dove si dominava la gran valle e le coste. Così mi piaceva la grossa collina, serpeggiante di schiene e di coste, nel buio. In passato era uguale, ma tanti lumi la punteggiavano, una vita tranquilla, uomini nelle case, riposo e allegrie. Anche adesso qualche volta si sentivano voci scoppiare, ridere in lontananza, ma il gran buio pesava, copriva ogni cosa, e la terra era tornata selvatica, sola, come l'avevo conosciuta da ragazzo (Pavese 2007: 5);

[...] il cielo dolce di febbraio apriva il cuore indolenzito. In collina, sotto le foglie fradice, dovevano spuntare i primi fiori. Mi ripromisi di cercarli (Pavese 2007: 84);

[...] seguirono giornate di vento che spazzavano i versanti, e di lassù si vedevano le creste successive, gli alberelli minuti, le case, i filari, fino ai boschi lontani (Pavese 2007: 117).

Il momento storico doloroso che fa da sfondo alla vicenda è segnalato da Pavese tramite l'uso di un coordinamento polisindetico fatto di frasi secche e brevi e lessico altamente connotante. Diamo alcuni assaggi: «la mattina rientrai con molta gente in città mentre ancora echeggiavano in lontananza schianti e boati. Dappertutto si correva e si portavano fagotti. L'asfalto dei viali era sparso di buche, di strati di foglie, di pozze d'acqua. Pareva avesse grandinato. Nella chiara luce crepitavano rossi e impudichi gli ultimi incendi» (Pavese 2007: 14); «le case sventrate fumavano. I crocicchi erano ingombri. In alto, tra i muri divelti, tappezzerie e lavandini pendevano al sole» ( Pavese 2007: 16); «Ma un colpo esplose, vicinissimo, in capo alla strada. Una raffica e un colpo. Poi urlacci, altri colpi di fuoco. I motori s'erano fermati; l'aria vibrava dei ronzii dolenti della pallottole» (Pavese 2007: 113).

Vidi il grosso autocarro. Lo vidi fermo, vuoto, per traverso. Una colata di benzina anneriva la strada, ma non era soltanto benzina. Lungo le ruote, davanti alla macchina, erano stesi corpi umani, e via via che mi avvicinavo la benzina arrossava [...] vidi sangue sui corpi. Uno - divisa grigioverde tigrata - era piombato sulla faccia, ma i piedi li aveva ancora sul camion. Usciva il sangue col cervello da sotto la guancia. Un altro, piccolo, le mani sul ventre, guardava in su, giallo, imbrattato. Poi altri contorti, accasciati, bocconi, di un livido sporco. Quelli distesi erano corti, un fagotto di cenci. Uno ce n'era in disparte sull'erba, che era saltato dalla strada per difendersi sparando: irrigidito ginocchioni contro il fildi- 
ferro, pareva vivo, colava sangue dalla bocca e dagli occhi, ragazzo di cera coronato di spine (Pavese 2007: 115).

In questo ultimo frammento, il narratore avvicina lentamente il lettore allo spettacolo sanguinoso: dapprima appare la benzina arrossata e il corpo steso tra la terra e il camion; segue la raccapricciante devastazione del corpo dell'uomo prima e degli altri «fagotti di cenci» poi; infine termina col «ragazzo di cera coronato di spine», novello Cristo morente. Questi inserti, come del resto le riflessioni e i ricordi del narratore, intervengono a spezzare il ritmo narrativo del racconto, quasi fossero una forza centrifuga che apre un ventaglio di motivi. A questa forza centrifuga si oppone però una forza centripeta che si manifesta in stilemi e lessemi che riportano l'attenzione del lettore sulla loro struttura sintagmatica e verbale. In molti casi l'andamento paratattico e la nominalità della frase provocano un'accelerazione del movimento narrativo e nello stesso tempo servono ad arginare la dispersione causata dagli inserti stessi.

Gli incipit dei diversi capitoli, impostati nella stragrande maggioranza su determinazioni temporali, nascondono un disegno compositivo aprioristico. Anche la concatenazione usata nel passaggio da un capitolo all'altro è il chiaro segnale di tale disegno. Si vedano questi casi:

- fine cap. IV: «Cate rincorse Dino che scappava, per portarlo a letto» inizio cap. V: «Cate uscì di nuovo nel cortile, le andai incontro»

- fine cap. XI: «Ma c'era anche un'altra notizia - che i nostri soldati scappavano e nessuno si sognava di resistere» inizio cap XII: «Alzai le spalle anche stavolta»

- fine cap. XXI: «Allora il vecchio si sedette, tenendo in mano la cavezza» inizio cap XXII: «Stemmo così molto tempo».

Questo progredire concatenato facilita l'interpretazione dei fatti da parte del lettore che viene così a essere ancor più partecipe e coinvolto. La tecnica della ripresa è alla fine e all'inizio del racconto, così come all'inizio e alla fine si situa la collina che, «non era un luogo tra gli altri, ma un aspetto delle cose, un modo di vivere» (Pavese 2007: 3) e «resta tuttora un paese d'infanzia, di falò, e di scappate, di giochi» ( pavese 2007: 121).

Tra le strutture semantiche che risaltano di più c'è il gioco contrastivo che è presente fin dall'apertura del romanzo tra collina e città. La collina, ambiente mitico che tutto racchiude (il ritorno all'infanzia con i falò, le scappate, i giochi, il rifugio dagli sconvolgimenti della guerra), fa da contraltare all'ambiente della città, ammutolita e raggelata dal «soprassalto notturno degli allarmi» (Pavese 2007: 3). In un crescendo significativo il romanzo le presenta entrambe e sembra che ora la guerra sia il motivo per legarle con una «risacca» di gente che trasmigra dall'una all'altra in cerca di rifugio e protezione. Lo spazio della storia è spaccato a metà: 
a) da una parte l'ambiente della collina:

«un terreno accidentato e serpeggiante, coltivato e selvatico» (p. 3)

«le stelle piovevano luce» (p. 7)

«sul fresco della collina, in quel vuoto, in quell'ansia che manteneva all'erta, ritrovavo un sapore più antico, contadino, remoto» (p. 8)

«a due passi dalla cresta avevo intorno delle schiene boscose» (p. 26)

«sotto alla fontana, nella conca di erbe grasse e fangose. Tra le piante apparivano buchi di cielo e aerei versanti. C'era in quel fresco un odore schiumoso, quasi salmastro» (p. 32)

«si cominciavano a vedere contadine per i campi, e le scalette contro i tronchi dei frutteti» (p. 56)

«le tendine bianche, fresche, si aprivano sulle foglie profonde e sul versante lontano dov'era un prato in mezzo ai boschi» (p. 65)

«la collina era bella, mostrava ormai la terra dura, polverulenta, nuda. Nei boschi s'incontravano giacigli scricchiolanti di foglie»» (p. 70)

«sui versanti svariavano vigneti e grano, freschi ancora di pioggia; non ricordavo così vive e così dolci colline» (p. 105)

«passavo sentieri, piccole forre, una croce di legno rizzata per voto. Il cielo altissimo era chiaro. A metà costa di quella collina, mi attendeva un gruppetto di case nitide» ( $\mathrm{p}$. 113) b) dall'altra quello della città:

«la città $[\ldots]$ tutta in fiamme e la gente morire» (p.3)

«la città raggelarsi, il trepestio, porte sbattersi, le vie sbigottite e deserte» (p. 7)

«Torino era tutta distrutta - ci sono migliaia di morti [...] hanno spianato la stazione, han bruciato i mercati [...] le case sventrate fumavano» (pp. 14-16)

«girai per Torino. Davvero sembrava l'indomani degli incendi. Era avvenuto qualcosa di enorme, un terremoto, cui soltanto i vecchi crolli e le macerie disseminati per le vie e riparati alla meglio, facevano adatto teatro» (p. 38)

«rintronarono due colpi, fragorosi sotto il portico; la gente cadde, si squagliò; tintinnarono i vetri in frantumi» (p.59)

«Torino era stata occupata [...] tedeschi ossuti e verdi come ramarri presidiavano la stazione, le caserme» (p. 62)

«metà della piazza era diroccata da bombe, qualche tedesco sfaccendato si aggirava e curiosava» (p. 84)

«da Torino, da tutto il Piemonte, quasi ogni giorno si parlava di condanne, di sevizie mai viste» (p. 97)

«una casetta sulla strada, annerita, sfondata, mi fermò e fece battere il cuore. Non vidi anima viva [...] pensai all'eco dei clamori, al sangue sparso, agli spari» (p. 109)

«la sparatoria era fitta e lamentosa. Quei tonfi sordi erano bombe a mano e scoppiavano attutiti. Gli schianti delle pallottole invece gemevano come voci di vivi» (pp. 113-114) 
Questo contrasto simbolico città-collina è paragonabile al contrasto guerra-pace, dove la seconda verrà raggiunta solo quando «tutti avremo accettato di far guerra. E allora forse avremo pace» (Pavese 2007: 121). In questo «forse» è racchiusa la disperazione del narratore, che non trova più serenità nemmeno camminando tra le colline perché «un tronco secco, un nodo d'erba, una schiena di roccia, mi paiono corpi distesi» (Pavese 2007: 122).

Come abbiamo detto, la scelta lessicale concorre a innescare l'azione centripeta del messaggio; a dimostrazione abbiamo scelto di fermare l'attenzione su una parola che è di altissima frequenza in questo testo: guerra. Questo lemma, che ricorre più volte in ogni capitolo, è un pedale ostinato che evidenzia la distruzione, la paura, l'orrore, il dolore. Si osservi questo schema in cui ne vengono riportate le occorrenze:

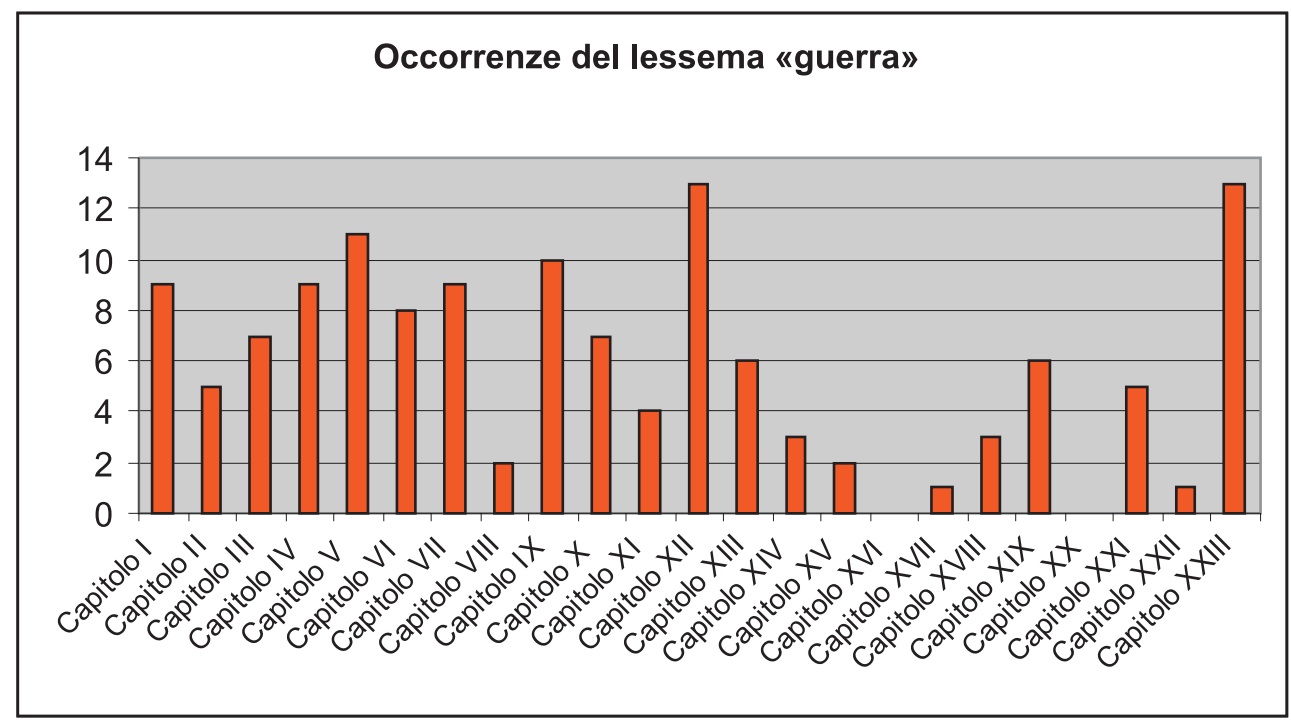

Il diagramma mostra che a capitoli in cui le occorrenze sono altissime seguono capitoli in cui compare poche volte (una, due o nessuna): ciò ha una spiegazione precisa. Non si parla di guerra nel capitolo VIII perché Cate e Corrado ragionano pacatamente sulla caduta del governo Mussolini e sulla conseguente speranza di pace; il capitolo XVI si occupa solo del rastrellamento nell'osteria Le Fontane; nel capitolo XVII si assiste alla descrizione della vita nel collegio di Chieri; nel capitolo XX il narratore inizia il fortunoso viaggio verso la casa paterna; infine il capitolo XXII propone una sola volta il sostantivo guerra, ma la narrazione ne evidenzia le conseguenze: la scena raccapricciante dei morti repubblichini, la gente che fa i fagotti, le stalle saccheggiate. Ė anche utile segnalare che la parola guerra è intimamente legata al campo semantico che la contraddistingue, a cominciare da battaglia, per continuare con sommosse, guerriglia, incursioni, stato d'assedio, eccidio, agguati, massacri, rovine, macerie, allarme, fuggiaschi, torture, paura, terrore, dolore, sangue, 
feriti, cadaveri, finimondo, morte, soldati, esercito, generali, maresciallo, pattuglie, truppa, divisione corazzata, paracadutisti, armi, rivoltella, fucili, pallottole, bossoli, bombe, spari, schianti, boati, raffiche, incendi, case sventrate crollate semidivelte. All'interno di questo campo troviamo le diverse tassonomie che riguardano le armi, le distruzioni, i soggetti della guerra; se vogliamo entrare ancora più nel particolare dei soggetti che combattono e si fronteggiano, al di sotto dell'iperonimo soldati troviamo partigiani, patrioti, tedeschi, fascisti, repubblichini.

Stilema caratteristico e ricorrente in questo romanzo è l'uso che l'autore fa di aggettivi o sostantivi messi in triade, comportamento che denota la cura per la ricerca della parola più connotante; del resto Pavese stesso aveva detto: «le parole sono tenere cose, intrattabili e vive, ma fatte per l'uomo e non l'uomo per loro. Sentiamo tutti di vivere in un tempo in cui bisogna riportare le parole alla solida e nuda nettezza di quando l'uomo le creava per servirsene» (Pavese 1968: 199).

«Le strade formicolavano di gente [...] indocile, credula e divertita» (3); «i tram stavano fermi, spalancati e deserti» (Pavese 2007: 14); «quel cauto equilibrio d'ansie, di attese e di futili speranze» (Pavese 2007: 50). Tale ricchezza lessicale fortemente evocativa arricchisce la referenzialità, dando vita a immagini reali, e la maggior parte di questi termini riguarda le distruzioni della guerra: «molta gente [...] barbuti, stracciati, affamati» (Pavese 2007: 63); «parlavano tutti di fame, di fughe, di guerra» (Pavese 2007: 103).

Lo spazio dell'orrore della guerra è spesso reso da similitudini, sinestesie e ossimori; di seguito diamo alcuni assaggi:

- «tutti i muri imbrattati come dall'ala impazzita di un uccello di fuoco» (Pavese 2007: 14); «una scheggia [...] tagliente e pesante come un ferro di zappa» (Pavese 2007: 25); «adesso fuggivo davvero, come fugge una lepre» (Pavese 2007: 107);

- «un tranquillo disordine» (Pavese 2007: 39); «un sorriso tagliente» (Pavese 2007: 66); «quella sua faccia molle» (Pavese 2007: 79); «la luce odorosa e fredda del mattino» (Pavese 2007: 101); «i ronzii dolenti delle pallottole» (Pavese 2007: 113);

- «incauto entusiasmo» (Pavese 2007: 39); «gli gridai sottovoce» (Pavese 2007: 114).

Lo schema del discorso è imperniato sulla figura della ripetizione: anafore, anadiplosi, epanalessi con la loro presenza ricca e multiforme rendono la paratassi coesa, donando chiarezza ed efficacia all'argomentazione, come è il caso del periodo quadrimembre di seguito citato, basato sulla ripetizione della negazione non:«non passa nessuno [...] la segretaria non si è ancora vista. Non si è visto Fellini. Non si può sapere niente» (Pavese 2007: 14) - che evidenzia la desolazione creata dal bombardamento notturno. Spesso le ripetizioni ricalcano la parlata quotidiana: «la guerra è la guerra» (Pavese 2007: 15); «ci sanno fare [...] ci sanno» (Pavese 2007: 59); «dov'era andato lo sapevo. Lo sapevo da un pezzo» (Pavese 2007: 100). In molti punti la sintassi è scarsamente sorvegliata e troviamo frasi sghembe del tipo: «sono i paesi che al mercato ci si va col fucile anche in tempo di pace» (Pavese 2007: 66); «era incredibile che gente come quella [...] avessero anche loro subito la guerra, la ventata, il terrore del mondo» (Pavese 2007: 109); «l'8 settembre ci sorprese che con Gregorio abbacchiavamo le noci» (Pavese 2007: 56). 
In La Casa in collina Pavese raggiunge un equilibrio tra la concretezza e la complessità del reale e il senso della componente simbolico-mitica. Nel romanzo la presenza della realtà storico-politica non impedisce di cogliere il senso che Pavese attribuiva al mito: «a un luogo, tra tutti, si dà un significato assoluto, isolandolo nel mondo [...] Così a ciascuno i luoghi dell'infanzia ritornano alla memoria; in essi accaddero cose che li han fatti unici e li trascelgono sul resto del mondo con questo suggello mitico» (Pavese 1974: 139). Egli dava al mito «la facoltà di reincarnarsi in ripetizioni che appaiono e sono creazioni ex novo. [Esso] vivrà nella coscienza come schema normativo dell'immaginazione affettiva» (Pavese 1974: 140). Come dice Giuditta Isotti Rosowsky «è questo schema, reinterpretato, completamente reinventato nel registro dell'attualità storica e politica, a fornire il disegno garante della coerenza del testo» (Isotti Rosowsky 2000: 91). In effetti più volte lungo il testo appaiono affermazioni che rivelano il compiacersi del narratore in ricordi d'infanzia, il suo interpretare la collina come un modo di vivere, un paese di falò e di giochi, un luogo dove nascondersi e guardare il cielo, dove «buttarsi nell'erba e giocare alla caccia o agli agguati» (Pavese 2007: 33), dove cercare protezione in un cespuglio e non «uscirne mai più» (Pavese 2007: 121).

\section{Bibliografia}

BARTHES, Roland (1973): S/Z. Una lettura di «Sarrasine» di Balzac. Torino, Einaudi. BERRUTO, Gaetano (1998): Sociolinguistica dell'italiano contemporaneo. Roma, Carocci. CANE, Eleonora (1969): Il discorso indiretto libero nella narrativa italiana del Novecento. Roma, Silva.

IsOTTI RoSOWSKY, Giuditta (2000): «Pavese: il romanzo deludente», in Esperienze letterarie, XXV, n. 3-4, pp. 87-101.

PAVESE, Cesare (1974): Feria d'agosto. Torino, Einaudi.

PAVESE, Cesare. (2007): La casa in collina. Torino, Einaudi.

PAVESE, Cesare (1971): La letteratura americana e altri saggi. Milano, Il Saggiatore.

PAVESE, Cesare (1968): Saggi letterari. Torino, Einaudi.

RoBBE-GRILLET, Alain (1961): «Natura, umanesimo, tragedia», in Una via per il romanzo futuro. Milano. $\mathrm{Ru}$ 